\title{
Differing responses of human stem cell-derived cardiomyocytes to arrhythmogenic drugs, determined using impedance measurements
}

\author{
Arisa Higa, Hirotaka Hoshi and Motoki Takagi \\ Medical-industrial Translational Research Center, Fukushima Medical University, \\ 1 Hikariga-oka, Fukushima, Fukushima 960-8031, Japan
}

(Received February 9, 2016; Accepted February 16, 2016)

\begin{abstract}
In recent years, human induced pluripotent stem cell-derived cardiomyocytes (hiPS$\mathrm{CMs}$ ) and human embryonic stem cell-derived cardiomyocytes (hES-CMs) have been widely used to develop cardiotoxicity assessment systems. To accurately evaluate the arrhythmogenic potential of drugs, we tested three types of hiPS-CMs (iCell Cardiomyocytes, Cellartis hiPS-CM, and Cor.4U) and a type of hES-CMs (Cellartis Pure hES-CM) using impedance technology. All CMs were cultured as confluent monolayers and their beating activity was analyzed with an impedance-based real-time monitoring instrument, the xCELLigence RTCA Cardio System. Measurement of impedance provided information about the $\mathrm{CM}$ beating rate and impedance amplitude between negative and positive peaks. Although all CMs except iCell CMs showed similar beating rates, their amplitudes were different. In addition, the patterns of contraction and relaxation were notably different between iCell CMs and the other CMs. The hiPSCMs and hES-CMs were treated with two arrhythmogenic drugs, sotalol and moxifloxacin, for $24 \mathrm{hr}$; the results showed that the drugs induced arrhythmic beating patterns on all CMs, although the response was varied. Thus, a more suitable type of CM remains to be found for optimal evaluation of the arrhythmogenic potential of drugs.
\end{abstract}

Key words: Arrhythmia, Cardiotoxicity, hiPS cell-derived cardiomyocytes, hES cell-derived cardiomyocytes, Impedance measurement, xCELLigence

\section{INTRODUCTION}

Unexpected cardiotoxicity is a significant concern in drug development and a major cause of drug withdrawal from the market. In the period between 1990 and 2001, eight drugs were withdrawn because of their arrhythmiainducing adverse effects (Fermini and Fossa, 2003). As a consequence, in vitro and in vivo assays to evaluate druginduced arrhythmia have been incorporated in the preclinical cardiac safety assessment of drugs. However, the effectiveness of these assays is limited by false positive and false negative results, differences in drug response between humans and the animal models used in preclinical phases, and the increasing incorporation of the three Rs of animal experimentation (replacement, refinement, and reduction) (Fermini and Fossa, 2003; Omata et al., 2005). To overcome these problems, human embryonic stem cell-derived cardiomyocytes (hES-CMs) and human induced stem cell-derived cardiomyocytes (hiPS$\mathrm{CMs}$ ) have been recently used for preclinical cardiac safety assessments, including evaluation of drug-induced arrhythmia (Yokoo et al., 2009; Liang et al., 2010; Sarić et al., 2014). hiPS-CMs are supplied by several manufacturers; the differential characteristics (e.g. growth, beating activity and drug response) under the same experimental conditions of these are unclear.

The assessment of arrhythmogenic potential of drugs has been based on the analysis of CM electrical activity using methodologies such as extracellular recording with sharp or patch electrodes (Honda et al., 2011), extracellular recording with multi-electrode arrays (Dick et al., 2010; Guo et al., 2011; Asakura et al., 2015), and optical imaging with voltage-sensitive dyes (Lopez-Izquierdo et al., 2014; Hayakawa et al., 2014). In addition, assessment by continuous monitoring of impedance measurements has been reported (Guo et al., 2011, 2013; Xi et al.,

Correspondence: Motoki Takagi (E-mail: m-takagi@fmu.ac.jp) 
2011; Himmel, 2013; Nguemo et al., 2012).

In our study, we measured impedance with a realtime monitoring instrument, the xCELLigence RTCA Cardio System (ACEA Bioscience, San Diego, CA, USA), to detect the beating activity of CMs. The xCELLigence system can be set in a culture incubator for labelfree determination of number, morphology, and attachment of cells in a 96-well plate for long periods of time (Xi et al., 2011). However, since the electrical activity of CMs cannot be measured directly with the xCELLigence system, the details of cellular mechanism in response to drugs are unclear. In addition, while there have been a number of reports regarding the evaluation of iCell Cardiomyocytes as hiPS-CMs with the xCELLigence system (Guo et al., 2011, 2013; Xi et al., 2011; Himmel, 2013; Nguemo et al., 2012; Talbert et al., 2015), comparative data for hiPS-CMs under the same experimental conditions have not yet been reported. Therefore, we evaluated the beating activities of iCell Cardiomyocytes, Cellartis hiPS-CM, and Cor.4U as hiPS-CMs, and Cellartis Pure hES-CM as hES-CMs with the xCELLigence system, and their response to arrhythmogenic drugs by measuring arrhythmia-like behavior.

\section{MATERIALS AND METHODS}

\section{Drugs and reagents}

$( \pm)$-Sotalol and moxifloxacin were purchased from LKT Laboratories (St. Paul, MN, USA) and AdooQ Bioscience (Irvine, CA, USA), respectively. All compounds were dissolved in a mixture of dimethyl sulfoxide (DMSO) and methanol $(7: 3)$ and stored at $-80^{\circ} \mathrm{C}$. Fibronectin, gentamicin, and Y-27632 used in the cell cultures were obtained from Wako Chemical (Tokyo, Japan).

\section{Cell culture and measurement of beating activity using the xCELLigence RTCA Cardio System}

iCell Cardiomyocytes and Cor.4U were purchased from Cellular Dynamics International (Madison, WI, USA) and Axiogenesis (Cologne, Germany), respectively. Cellartis hiPS-CM and Pure hES-CM were obtained from Takara Bio (Shiga, Japan). These CMs were cultured according to the manufacturer's protocol.

iCell CMs were seeded onto fibronectin coated 6-well plates $(10 \mu \mathrm{g} /$ well $)$ at a density of $7.5 \times 10^{5}$ cells/well, and maintained in the plating medium at $37^{\circ} \mathrm{C}$ in a humidified incubator with $7 \% \mathrm{CO}_{2}$. The plating medium was replaced by a maintenance medium supplemented with gentamicin $(50 \mu \mathrm{g} / \mathrm{mL})$ at $48 \mathrm{hr}$ after seeding. The medium was changed every other day until day 6 after seeding.
Cellartis hiPS-CMs and hES-CMs were seeded onto fibronectin coated 6 -well plates $(75 \mu \mathrm{g} /$ well) at a density of $6 \times 10^{5}$ cells/well and maintained in a thawing medium supplemented with $20 \%$ fetal bovine serum (FBS) and $10 \mu \mathrm{M} \mathrm{Y}-27632$ at $37^{\circ} \mathrm{C}$ in a humidified incubator with $5 \% \mathrm{CO}_{2}$. The thawing medium was replaced by a culture medium supplemented with FBS (10\%) and gentamicin $(50 \mu \mathrm{g} / \mathrm{mL})$ at $48 \mathrm{hr}$ after seeding. The medium was changed every other day until day 6 after seeding.

The iCell and Cellartis CMs were dissociated from the 6-well plates 7 days after seeding and reseeded into the E-plate 96 (ACEA Bioscience), which is a special singleuse 96-well plate for performing cell-based assays in the xCELLigence RTCA Cardio System. Wells were coated with fibronectin $(0.5 \mu \mathrm{g} /$ well for iCell and $2.5 \mu \mathrm{g} /$ well for Cellartis) and the CMs were seeded at a density of $4 \times$ $10^{4}$ cells/well. The plates were assessed with the xCELLigence RTCA Cardio System at $37^{\circ} \mathrm{C}$ in a humidified incubator following the manufacturer's protocol. The impedance was monitored every $60 \mathrm{~min}$ for $20 \mathrm{sec}$. The medium was replaced at $48 \mathrm{hr}$ after reseeding. The next day, the CMs were treated with the drugs.

Cor.4U CMs were directly seeded onto an E-plate 96 coated with fibronectin $(0.5 \mu \mathrm{g} /$ well $)$ at a density of $4 \times 10^{4}$ cells/well, and the plate was placed into the $\mathrm{xCEL}$ Ligence system at $37^{\circ} \mathrm{C}$ in a humidified incubator with $5 \% \mathrm{CO}_{2}$. The impedance was monitored every $60 \mathrm{~min}$ for $20 \mathrm{sec}$. The culture medium was replaced with a medium supplemented with gentamicin $(50 \mu \mathrm{g} / \mathrm{mL})$ at 16 and $24 \mathrm{hr}$ after seeding. Thereafter, the medium was replaced once every day until addition of the drugs. The CMs were treated with the drugs after $72 \mathrm{hr}$.

\section{Measurement of the drug response using xCELLigence RTCA Cardio System}

The beating activity, amplitude, and beating rhythm irregularity (BRI) of CMs were measured after seeding into the E-plate 96. When the conditions of beating rate, amplitude, and BRI satisfied the criteria of the manufacturer's protocol $(30-65 / \mathrm{min},>0.03$, and $<10 \%$, respectively), the CMs were treated with the drugs. The medium in the plate was replaced and the plate was loaded on the $\mathrm{xCELLigence} \mathrm{system} \mathrm{for} 4 \mathrm{hr}$. The hiPS-CMs and hESCMs were then treated with sotalol $(100 \mu \mathrm{M})$ and moxifloxacin $(100 \mu \mathrm{M})$ for $24 \mathrm{hr}$. The vehicle solvent was used as a control in all experiments at a concentration of $0.1 \%$. After drug treatment, the impedance of CMs was measured every minute for the first hour, every $2 \mathrm{~min}$ for the second hour, every $5 \mathrm{~min}$ for the third hour, and every $30 \mathrm{~min}$ from 4-24 hr. The data were analyzed using RTCA Cardio Software Ver. 1.0. 
Differing responses among cardiomyocytes to arrhythmogenic drugs

\section{RESULTS AND DISCUSSION}

To develop our evaluating system for arrhythmogenic potential, we measured the beating rate and amplitude of three types of hiPS-CMs (iCell Cardiomyocytes, Cellartis hiPS-CM, and Cor.4U) and one type of hES-CMs (Cellartis Pure hES-CM) by measuring impedance with the xCELLigence system. This system measures the impedance signal and then processes and displays the data by converting impedance into Cell Index values (Xi et al., 2011). In addition, it can continually record the changes in adhesion, spreading and beating activity of the CMs. The dynamic change of the Cell Index values of iCell CMs measured by the xCELLigence system is shown in Fig. 1A. This change indicates the attachment, growth, and beating activity of iCell CMs over time. The Cell Index value rapidly increased for the first $3 \mathrm{hr}$ after seeding and continued to increase gradually thereafter. The value finally became stable at $48 \mathrm{hr}$ after seeding. These results implied that the CMs firmly attached and spread out on the wells. The beating period and amplitude of iCell CMs at $72 \mathrm{hr}$ after seeding are shown in Fig. 1B. The impedance trace lasted for $9 \mathrm{sec}$. The distance between negative peaks in the $\mathrm{x}$-axis indicates the beating period, and the difference in Cell Index values between negative and positive peaks indicates the amplitude. The decrease and increase of Cell Index values represent the CMs contraction and relaxation, respectively. These cycles of the CMs were detected by the subtle change of Cell Index values.

Since culture plates coating material affects $\mathrm{CM}$ beating patterns (gelatin or fibronectin) (Guo et al., 2011, 2013; Xi et al., 2011; Himmel, 2013; Nguemo et al., 2012; Talbert et al., 2015), in this study all four types of $\mathrm{CMs}$ were cultured in fibronectin-coated plates to ensure consistency. The CMs were cultured as a confluent monolayer on 60 wells of E-plate 96 and displayed spontaneous and stable beating. We repeated the experiments for all CMs and observed this behavior with sufficient reproducibility. The differences in beating rate and amplitude between iCell, Cellartis, and Cor.4U hiPS-CMs, and hES$\mathrm{CMs}$ was measured with the $\mathrm{xCELLigence} \mathrm{system} \mathrm{and}$ the data from ten wells of each CM type were analyzed. The beating profiles of each $\mathrm{CM}$ at 3-72 $\mathrm{hr}$ after seeding into the E-plate 96 are shown in Fig. 2A. iCell CMs in four wells out of ten started beating $3 \mathrm{hr}$ after seeding. The beating rate and amplitude subsequently became stable at $36 \mathrm{hr}$ (Fig. 2), and the BRI was 2.8\%. On the other hand, the beating of Cellartis and Cor.4U hiPS-CMs, and the hES-CMs started at $24 \mathrm{hr}$ after seeding in several of the 10 wells. However, no synchronized beating was detected and the BRI was not calculated by the xCELLigence system. Their beating rate and amplitude became

A
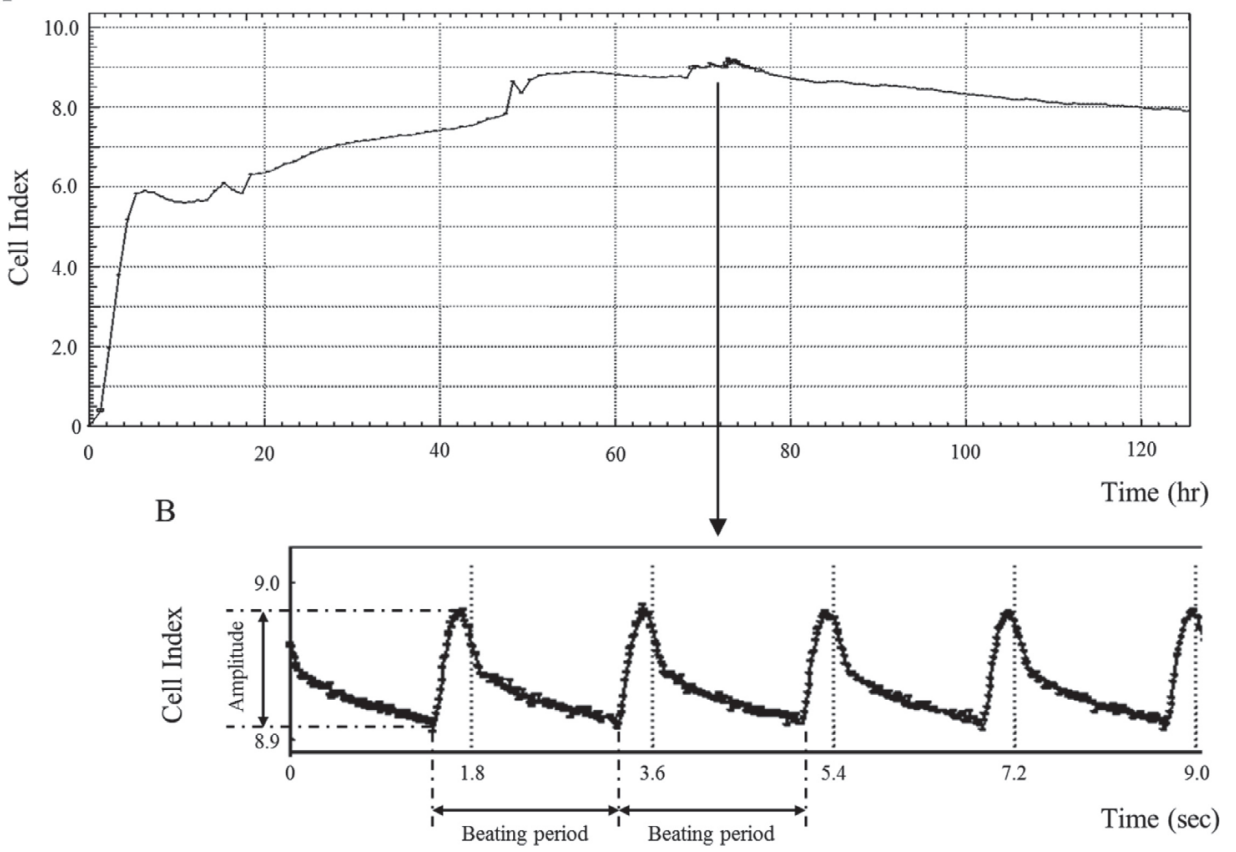

Fig. 1. (A) Impedance measurement of beating activity of iCell CMs with the xCELLigence RTCA Cardio System. (B) The beating period and amplitude of iCell CMs $72 \mathrm{hr}$ after seeding. 
A. Higa et al.
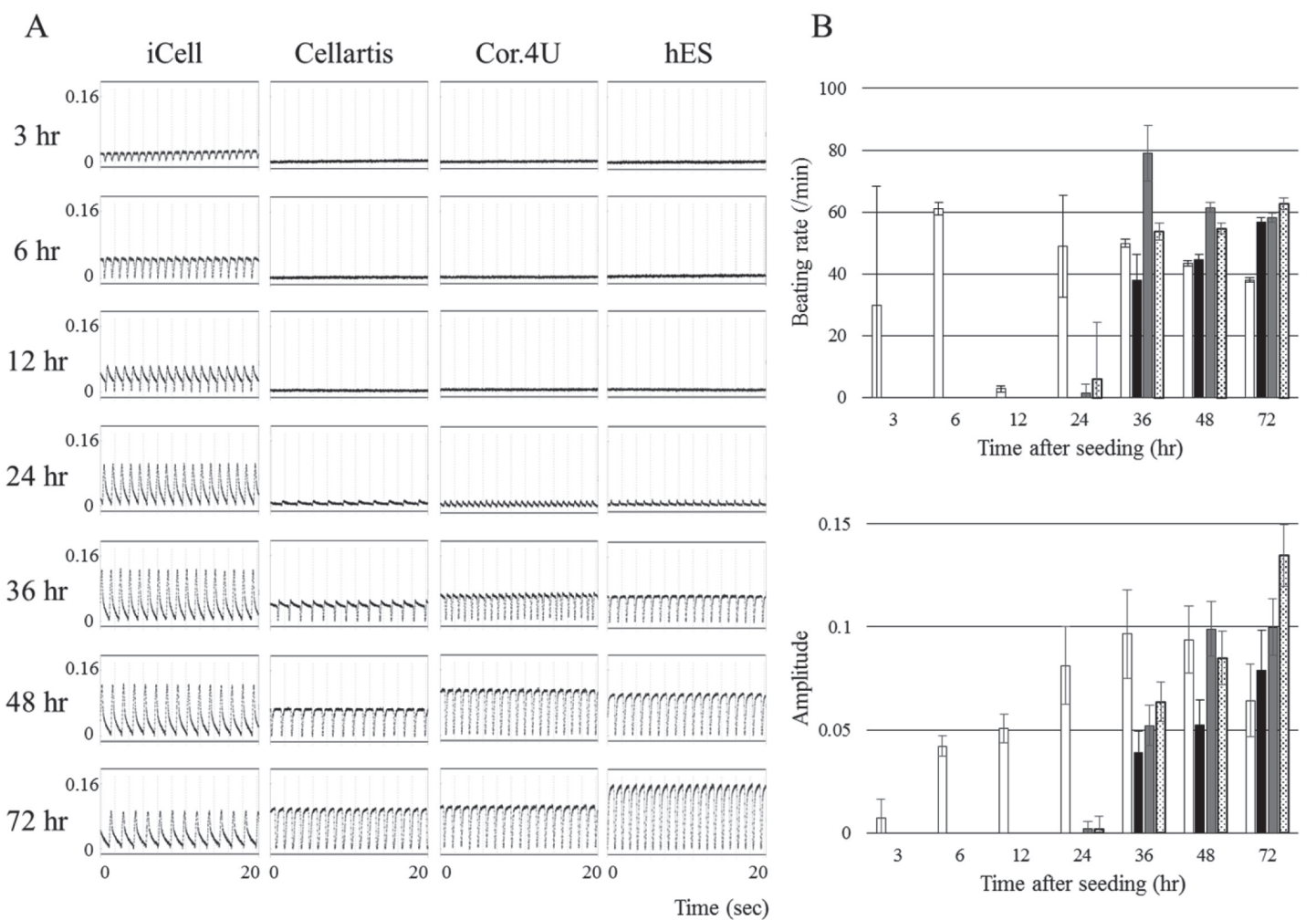

Fig. 2. The beating activities of three types of hiPS-CMs and a type of hES-CMs with the xCELLigence RTCA Cardio System (A) Impedance measurements changes in time since seeding onto the E-plates. (B) Beating rates and amplitudes of CMs over time. White bar is iCell, black bar is Cellartis hiPS-CMs, gray bar is Cor.4U, and dot bar is Cellartis hES-CMs. Data represent mean \pm S.D. $(n=10)$.

stable (BRI; 1.1\%) at $48 \mathrm{hr}$ (Fig. 2). The variance of beating rates and amplitudes of all CMs converged about 72 $\mathrm{hr}$ after seeding and all BRI values were $<2.5 \%$ (Fig. 2B). The beating rate of iCell CMs was about 40 times/min at $72 \mathrm{hr}$. The beating rates of hiPS-CMs and hES-CMs were about 60 times/min at $72 \mathrm{hr}$. The beating rate of iCell CMs appeared to be slightly lower than those of Cellartis hiPS-CMs and Cor.4U CMs. By contrast, the amplitude was different across the different types of CMs. The amplitude of iCell CMs was 0.1 at $36-48 \mathrm{hr}$ but decreased by 0.06 at $72 \mathrm{hr}$. The amplitudes of Cellartis hiPS-CMs and Cor.4U CMs, and hES-CMs at $72 \mathrm{hr}$ were $0.08,0.10$, and 0.13 , respectively. Thus, the amplitude of hES-CMs was the biggest among all CMs. The most important difference between these CMs was the pattern of the contraction and relaxation cycle. The relaxation period of iCell CMs was very short and the contraction period was long, whereas the relaxation periods of the other CMs were long. The pattern of iCell CMs was identical to the previously reported using fibronectin-coated plates (Xi et al.,
2011; Nguemo et al., 2012; Talbert et al., 2015). Overall, these results indicate that the characteristics of iCell CMs are notably different from those of the other CMs; however, there was no significant difference in beating activity between Cellartis hiPS-CMs and hES-CMs. Although the reasons for these differences are unclear, they may be due to the characteristics of the original hiPS and hES cells and the differentiation strategy from hiPS and hES cells to CMs.

To evaluate the response of the four types of CMs to drugs, we selected two arrhythmogenic drugs, sotalol and moxifloxacin. Sotalol is a cardiac drug that is used to treat some specific types of arrhythmia. Moxifloxacin is a synthetic fluoroquinolone used as an antibacterial agent. These two drugs have been reported to clinically induce torsades de pointes (TdP) through prolongation of the QT interval (the Japan Pharmaceutical References). Sotalol is known to induce arrhythmia frequently, whereas the clinical occurrence of arrhythmia induced by moxifloxacin is low. We tested the response of CMs to these two drugs at 
Differing responses among cardiomyocytes to arrhythmogenic drugs

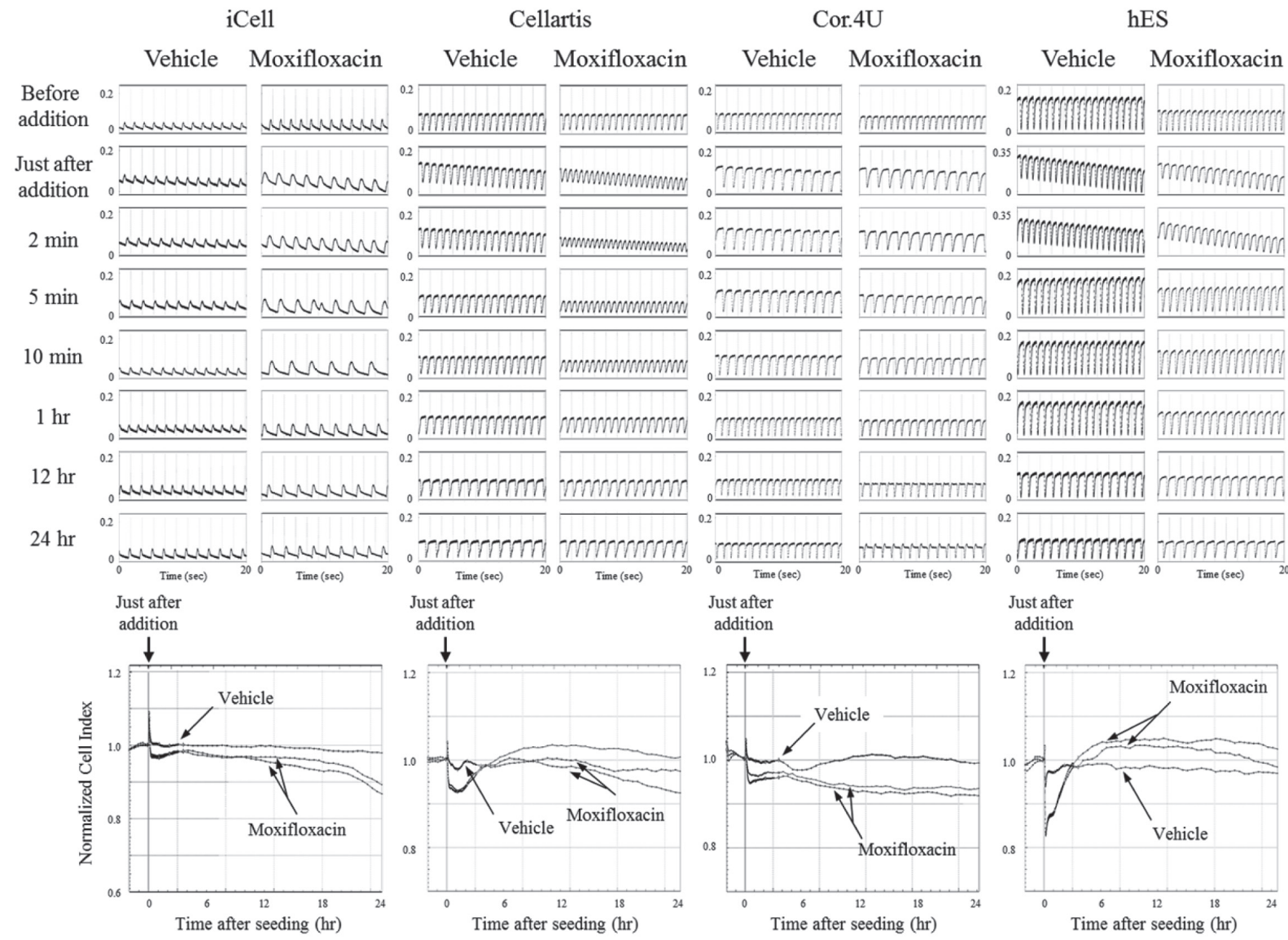

Fig. 3. Responses of CMs to moxifloxacin. The upper part shows the beating activities of hiPS-CMs and hES-CMs. The time shown is the time passed after the addition of drugs. The lower part shows the dynamic changes of Cell Index. The data are normalized with the values before drug addition and indicates the values of 2 wells of the E-plate 96.

$72 \mathrm{hr}$ after seeding onto the E-plate 96. The CMs were then evaluated by the xCELLigence system for $24 \mathrm{hr}$. The treated concentrations of moxifloxacin and sotalol were determined by our preliminary examinations and a previous report (Guo et al., 2013). The beating activities of all CMs did not change when the cells were treated with the vehicle (mixture of DMSO and methanol) (Figs. 3 and 4). As predicted, treatment with arrhythmogenic drugs resulted in a disturbance of the beating rhythm. We confirmed by microscope that sotalol and moxifloxacin had no cytotoxic effect on any of the CMs, even after $24 \mathrm{hr}$ of exposure (data not shown). In fact, the Cell Index values of all CMs were almost the same before and after the treatment with moxifloxacin, indicating that this drug induced arrhythmogenic activity without cytotoxicity (Fig. 3, lower part). On the other hand, the Cell Index values of Cor.4U CMs treated with sotalol decreased (Fig. 4, lower part). Thus, sotalol may induce weak cytotoxicity in

\section{Cor.4U CMs.}

The drug response of CMs to moxifloxacin was varied (Fig. 3). Moxifloxacin induced a change in beating activity of iCell CMs at a concentration of $100 \mu \mathrm{M}$ in our preliminary examinations (data not shown). The beating rate of iCell CMs was reduced by moxifloxacin with no effects on amplitude. The beating rate of Cellartis hiPS-CMs strongly increased for $10 \mathrm{~min}$ and subsequently decreased. The amplitude decreased for $1 \mathrm{hr}$, but then returned to its condition before treatment with the drug. The beating rate of Cor.4U CMs also decreased and the amplitude increased for $10 \mathrm{~min}$. Moreover, abnormal beating patterns were detected from $12 \mathrm{hr}$ onwards. The beating rate of hES-CMs gradually decreased.

It has been reported that sotalol caused arrhythmic beating of iCell CMs at a concentration of $100 \mu \mathrm{M}$ (Guo et al., 2013); therefore, we also chose this concentration for our experiment. Sotalol induced a typical 
A. Higa et al.

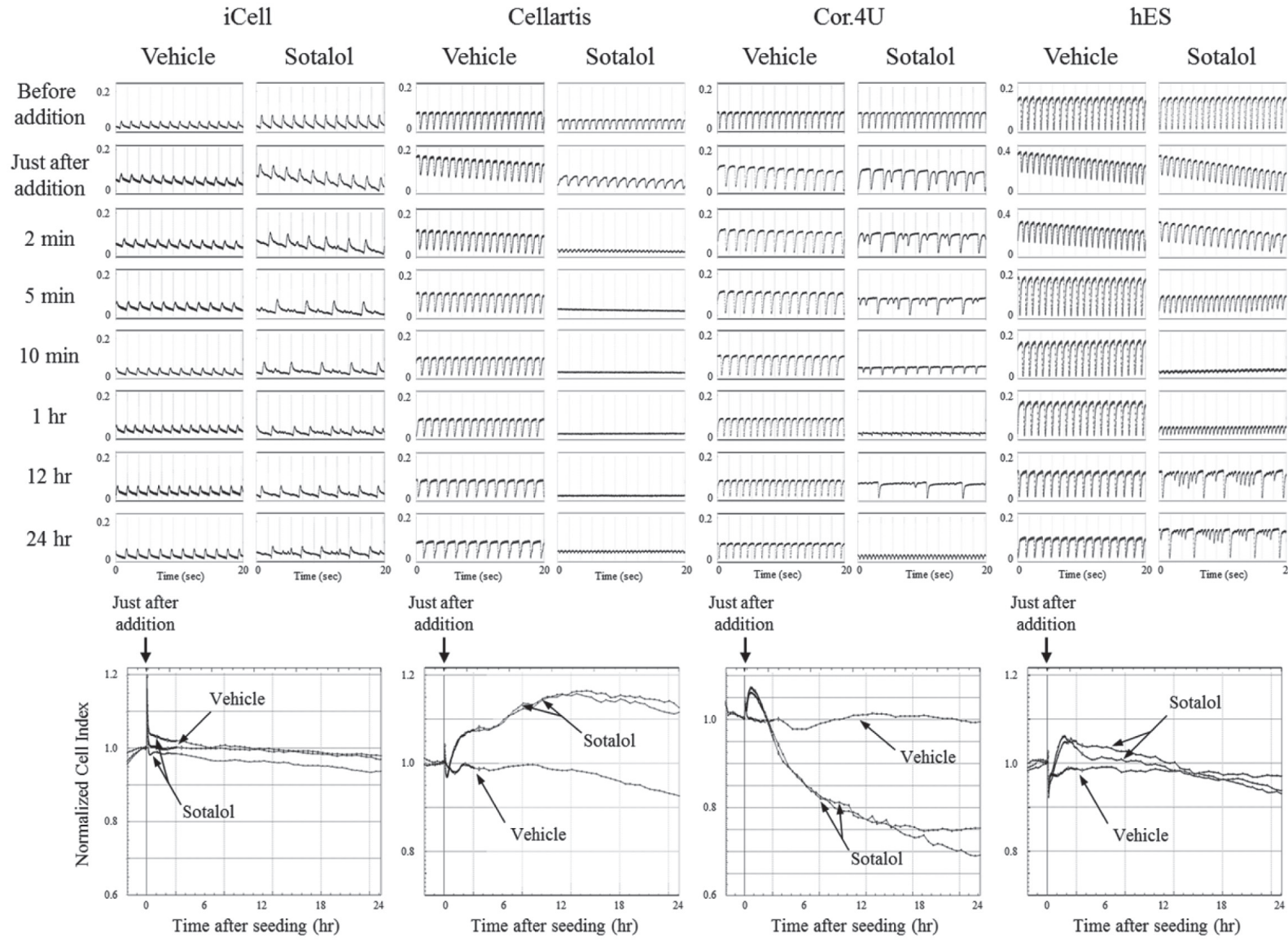

Fig. 4. Responses of CMs to sotalol. The upper part shows the beating activities of hiPS-CMs and hES-CMs. The time shown is the time passed after the addition of drugs. The lower part shows the dynamic changes of Cell Index. The data are normalized with the values before drug addition and indicates the values of 2 wells of the E-plate 96.

arrhythmic beating pattern on all CMs (Fig. 4). The beating rates of all hiPS-CMs decreased immediately after treatment with sotalol. The abnormal beating rhythm was sustained for $24 \mathrm{hr}$ in iCell and Cor.4U CMs. The abnormal beating rhythm in iCell CMs with fibronectin-coated plates was similar to a previous report (Nguemo et al., 2012). Since the dynamic Cell Index values of Cor.4U $\mathrm{CMs}$ decreased, the CMs were treated with a concentration of $10 \mu \mathrm{M}$. However, the Cell Index values and beating rhythm of Cor.4U CMs showed no change (data not shown). The beating activity of Cellartis hiPS-CMs arrested at 5 min after addition of the drug. Since the dynamic Cell Index values of Cellartis hiPS-CMs increased, the CMs arrested on relaxation cycle of the CMs. In contrast, sotalol caused increase of the hES-CMs beating rate and although the beating discontinued 10 min after drug treatment, it recovered after $1 \mathrm{hr}$. The amplitude of iCell and Cor.4U CMs and the hES-CMs decreased with time.
In conclusion, these results indicate that although arrhythmogenic activity can be evaluated with these CMs, they display diverse beating behaviors and response patterns in the presence of arrhythmogenic drugs. In addition, sotalol, a strong arrhythmogenic agent, was shown to induce more dramatic changes to the beating activity of CMs than moxifloxacin. Therefore, we could not select the most suitable CMs to evaluate the arrhythmogenic potential of drugs. Further studies are currently underway to examine in detail the response of CMs to drugs, to allow for accurate predictions or their arrhythmogenic potential in preclinical cardiac safety assessment of drugs.

\section{ACKNOWLEDGMENTS}

This research was supported by grants for translational research programs from Fukushima Prefecture. 
Differing responses among cardiomyocytes to arrhythmogenic drugs

Conflict of interest---- The authors declare that there is no conflict of interest.

\section{REFERENCES}

Asakura, K., Hayashi, S., Ojima, A., Taniguchi, T., Miyamoto, N., Nakamori, C., Nagasawa, C., Kitamura, T., Osada, T., Honda, Y., Kasai, C., Ando, H., Kanda, Y., Sekino. Y. and Sawada, K. (2015): Improvement of acquisition and analysis methods in multi-electrode array experiments with iPS cell-derived cardiomyocytes. J. Pharmacol. Toxicol. Methods., 75, 17-26.

Dick, E., Rajamohan, D., Ronksley, J. and Denning, C. (2010): Evaluating the utility of cardiomyocytes from human pluripotent stem cells for drug screening. Biochem. Soc. Trans., 38, 10371045.

Fermini, B. and Fossa, A.A. (2003): The impact of drug-induced QT interval prolongation on drug discovery and development. Nat. Rev. Drug Discov., 2, 439-447.

Guo, L., Abrams, R.M., Babiarz, J.E., Cohen, J.D., Kameoka, S. Sanders, M.J., Chiao, E. and Kolaja, K.L. (2011): Estimating the risk of drug-induced proarrhythmia using human induced pluripotent stem cell-derived cardiomyocytes. Toxicol. Sci., 123, 281-289.

Guo, L., Coyle, L., Abrams, R.M., Kemper, R., Chiao, E.T. and Kolaja, K.L. (2013): Refining the human iPSC-cardiomyocyte arrhythmic risk assessment model. Toxicol. Sci., 136, 581-594.

Hayakawa, T., Kunihiro, T., Ando, T., Kobayashi, S., Matsui, E., Yada, H., Kanda, Y., Kurokawa, J. and Furukawa, T. (2014): Image-based evaluation of contraction-relaxation kinetics of human-induced pluripotent stem cell-derived cardiomyocytes: Correlation and complementarity with extracellular electrophysiology. J. Mol. Cell Cardiol., 77, 178-191.

Himmel, H.M. (2013): Drug-induced functional cardiotoxicity screening in stem cell-derived human and mouse cardiomyocytes: effects of reference compounds. J. Pharmacol. Toxicol. Methods, 68, 97-111.

Honda, M., Kiyokawa, J., Tabo, M. and Inoue, T. (2011): Electrophysiological characterization of cardiomyocytes derived from human induced pluripotent stem cells. J. Pharmacol. Sci., 117, 149-159.

Liang, H., Matzkies, M., Schunkert, H., Tang, M., Bonnemeier, H., Hescheler, J. and Reppel, M. (2010): Human and murine embryonic stem cell-derived cardiomyocytes serve together as a valuable model for drug safety screening. Cell Physiol. Biochem., 25, 459-466.

Lopez-Izquierdo, A., Warren, M., Riedel, M., Cho, S., Lai, S., Lux, R.L., Spitzer, K.W., Benjamin, I.J., Tristani-Firouzi, M. and Jou, C.J. (2014): A near-infrared fluorescent voltage-sensitive dye allows for moderate-throughput electrophysiological analyses of human induced pluripotent stem cell-derived cardiomyocytes. Am. J. Physiol. Heart Circ. Physiol., 307, H1370-1377.

Nguemo, F., Šarić, T., Pfannkuche, K., Watzele, M., Reppel, M. and Hescheler, J. (2012): In vitro model for assessing arrhythmogenic properties of drugs based on high-resolution impedance measurements. Cell Physiol. Biochem., 29, 819-832.

Omata, T., Kasai, C., Hashimoto, M., Hombo, T. and Yamamoto, K. (2005): QT PRODACT: comparison of non-clinical studies for drug-induced delay in ventricular repolarization and their role in safety evaluation in humans. J. Pharmacol. Sci., 99, 31-41.

Sarić, T., Halbach, M., Khalil, M. and Er, F. (2014): Induced pluripotent stem cells as cardiac arrhythmic in vitro models and the impact for drug discovery. Expert Opin. Drug Discov., 9, 55-76.

Talbert, D.R., Doherty, K.R., Trusk, P.B., Moran, D.M., Shell, S.A. and Bacus, S. (2015): A multi-parameter in vitro screen in human stem cell-derived cardiomyocytes identifies ponatinib-induced structural and functional cardiac toxicity. Toxicol. Sci., 143, 147-155.

Xi, B., Wang, T., Li, N., Ouyang, W., Zhang, W., Wu, J., Xu, X., Wang, X. and Abassi, YA. (2011): Functional cardiotoxicity profiling and screening using the xCELLigence RTCA Cardio System. Assay Drug Dev. Technol., 9, 415-421.

Yokoo, N., Baba, S., Kaichi, S., Niwa, A., Mima, T., Doi, H., Yamanaka, S., Nakahata, T. and Heike, T. (2009): The effects of cardioactive drugs on cardiomyocytes derived from human induced pluripotent stem cells. Biochem. Biophys. Res. Commun., 387, 482-488. 